\title{
Dichorionic twins discordant for intrauterine growth retardation
}

\author{
N J Sebire, C D’Ercole, K Hughes, J Rennie, K H Nicolaides
}

\begin{abstract}
A policy of expectant management until 32 weeks of gestation in 29 dichorionic pregnancies discordant for growth retardation resulted in an overall mortality of $24 \%$ (95\% CI $13.9-37.2 \%)$ and a handicap of $2.2 \%(95 \%$ CI $0-12.0 \%)$. None of the normally grown co-twins died or was handicapped as a result of iatrogenic prematurity.
\end{abstract}

(Arch Dis Child 1997;77:F235-F236)

Keywords: twins; dichorionic pregnancy; handicap; intrauterine growth retardation

In singleton pregnancies with fetal growth retardation due to presumed utero-placental insufficiency the aim of antenatal care is to select the appropriate time for delivery. This is achieved by balancing the relative risks of intrauterine death with expectant management and neonatal death or handicap from iatrogenic preterm delivery. In dichorionic twin pregnancies discordant for intrauterine growth retardation the condition of both fetuses needs to be considered. This study reports the results of our policy of managing such pregnancies expectantly by avoiding iatrogenic preterm delivery, irrespective of the condition of the smaller twin, unless the minimum gestation was 32 weeks and /or the estimated fetal weight of the larger twin was more than $1500 \mathrm{~g}$ in 29 dichorionic pregnancies discordant for intrauterine growth retardation.

\section{Methods}

The Harris Birthright Research Centre is a referral unit for fetal diagnosis and treatment. Details on patients' demographic characteristics, medical and obstetric history, and ultrasound findings are entered into a computer database at the time of the ultrasound examination. Twin pregnancies were classified as dichorionic if the placentas were not adjacent to each other or the lambda sign was present. ${ }^{12}$ The results of any investigations and pregnancy outcome were entered into the database as they became available.

A computer search of the database was made for dichorionic twin pregnancies discordant for fetal growth retardation in the absence of a fetal abnormality or chromosomal defect presenting at 24-32 weeks of gestation from 1986-96. The individual files of these pregnancies were examined to determine survival rates and the general practitioners were contacted when the babies were at a median age of 28 months (range 11-118 months), to ascertain if the child was developing normally or had any major disability, including cerebral palsy, deafness, blindness or developmental delay.

\section{Results}

Twenty nine dichorionic twin pregnancies discordant for fetal growth retardation were identified. The median gestation at presentation was 28 (range 24-32) weeks and the median maternal age was 31 (range 16-41) years. In 27 cases there was no obvious maternal cause for the growth restriction, but there was one case of maternal sickle cell disease and another of pre-eclampsia (treated with Labetalol). In all cases one of the fetuses was appropriately grown for gestational age with normal amniotic fluid volume and biophysical profile. In contrast, the growth retarded co-twin had reduced amniotic fluid volume in $20(69 \%)$ cases, and in $22(76 \%)$ cases there were abnormalities in the umbilical artery Doppler waveform. The ultrasonographically estimated fetal weight ${ }^{3}$ at presentation in each fetus is shown in fig $1 .^{4}$ The estimated weight of the growth retarded fetus was below $500 \mathrm{~g}$ in $11(38 \%)$ cases, 500$750 \mathrm{~g}$ in seven $(24 \%)$ cases, $751-1000 \mathrm{~g}$ in five $(17 \%)$ cases, $1001-1250 \mathrm{~g}$ in four $(14 \%)$ cases and $>1250 \mathrm{~g}$ in two $(7 \%)$ cases.

All pregnancies were managed expectantly to avoid iatrogenic preterm delivery until the minimum gestation was 32 weeks or the estimated fetal weight of the larger twin was more than $1500 \mathrm{~g}$. Two cases delivered spontaneously at 27 and 28 weeks of gestation. The median gestational age at delivery was 33 weeks (range 27-39 weeks).

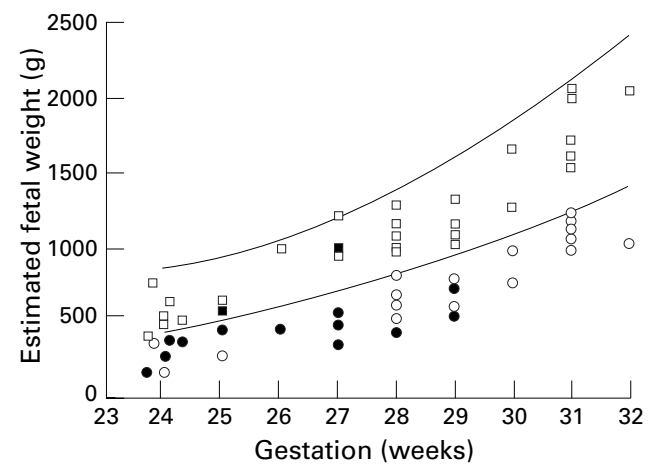

Figure 1 Estimated weight of each fetus in 29 dichorionic twin pregnancies at the time of referral, plotted on the appropriate normal range for singletons (mean, 5th, and 95th centiles). ${ }^{4}$ The squares represent the values for the appropriately grown fetuses and the circles for the growth retarded ones. Open squares and circles represent the survivors and closed squares or circles represent those that died either in utero or in the neonatal period. 
There were 44 survivors and $14(24 \%)$ perinatal deaths. In 10 cases the severely growth retarded twin died in utero at 24-32 weeks (median 29 weeks) of gestation, but the appropriately grown co-twin survived following delivery at 30-39 weeks (median 35 weeks) of gestation. The median birthweight of the growth retarded fetus was $900 \mathrm{~g}$ (range $480-2410 \mathrm{~g}$ ) and of the normally grown twin was $2040 \mathrm{~g}$ (range $660-3090 \mathrm{~g}$ ). Both fetuses from the pregnancies that delivered spontaneously at 27-28 weeks of gestation died in the neonatal period. Among the survivors 43 infants were developmentally normal at 2 years of age. However, one growth retarded fetus who was delivered at 32 weeks of gestation weighing $900 \mathrm{~g}$ had severe developmental delay, spastic diplegia, and blindness. The overall mortality was therefore $24 \%$ (95\% CI $13.9-37.2 \%)$ and the risk of handicap in survivors, $2.2 \%(95 \%$ CI $0-12.0 \%)$. The mortality and handicap rates of the normally grown twin were $6.9 \%(95 \%$ CI $0.8-22.8 \%)$ and $0 \%(95 \%$ CI $0-14.6 \%)$, respectively, and for the growth retarded twin, 41.4\% (95\% CI 23.5-61.1\%) and $5.9 \%$ (95\% CI $0.1-28.7 \%)$, respectively.

\section{Discussion}

The policy of expectant management until 32 weeks or an estimated fetal weight of the larger fetus of $1500 \mathrm{~g}$ aimed at minimising the risk of postnatal death and handicap of the appropriately grown fetus, by avoiding iatrogenic severe preterm delivery irrespective of the condition of the growth retarded co-twin. After 32 weeks the timing of delivery aimed to maximise the chance of survival of the growth retarded twin. Apart from two pregnancies that spontaneously delivered at 27-28 weeks, resulting in the death of all four babies, in all other cases the appropriately grown twin survived with no handicap. However, in $35 \%$ of pregnancies the growth retarded fetus died in utero.

An alternative policy would be to manage such pregnancies as if they were singletons with a growth retarded fetus. The minimum gestation at which the growth retarded fetus could potentially survive is about 26 weeks, but data from singleton pregnancies suggest that iatrogenic delivery at this gestation would be associated with an estimated risk of death in $40 \%$ of the appropriately grown, and $70 \%$ of the growth retarded babies; furthermore, about $25 \%$ of the appropriately grown and about $70 \%$ of the growth retarded survivors would potentially be severely handicapped. ${ }^{5}$

The policy of expectant management is only applicable to dichorionic pregnancies because intrauterine death of the growth retarded fetus is associated with minimal risk of death or handicap to the healthy twin. ${ }^{6}$ In contrast, in monochorionic pregnancies death of one fetus often results in death or neurological sequelae for the co-twin because of severe hypotension due to fetal haemorrhage into the dead feto-placental unit through the communicating placental vessels. ${ }^{6}$

1 Sepulveda W, Sebire NJ, Hughes K, Odibo A, Nicolaides $\mathrm{KH}$. The lambda sign at 10-14 weeks of gestation as a predictor of chorionicity in twin pregnancies. Ultrasound Obstet Gynecol 1996;7: 421-3.

2 Bessis R, Papiernik E. Echographic imagery of amniotic membranes in twin pregnancies. In: Gedda L, Parisi P, eds. Twin research 3: Twin biology and multiple pregnancy. New York: Alan R. Liss, 1981:183-7.

3 Hadlock FP, Harrist RB, Sharman RS. Estimation of fetal weight by ultrasound. Am f Obstet Gynecol 1985;151:333-

4 Yudkin PL, Aboulfa M, Eyre JA, Redman CWG, Wilkinson AR. New birthweight and head circumference centiles for
gestational ages 24-42 weeks. Early Hum Dev 1987;15:4552 .

5 Rennie JM. Perinatal management at the lower margin of viability. Arch Dis Child 1996; 74: F214-18.

6 Murphy KW. Intrauterine death in a twin: implications for the survivor. In: Ward RH, Whittle M, eds. Multiple Pregnancy. London: RCOG Press, 1995:218-30. 